\title{
Performance Analysis of Uplink SCMA With Receiver Diversity and Randomly Deployed Users
}

DOI:

10.1109/TVT.2017.2728607

Document Version

Accepted author manuscript

Link to publication record in Manchester Research Explorer

\section{Citation for published version (APA):}

Bao, J., Ma, Z., Xiao, M., Ding, Z., \& Zhu, Z. (2018). Performance Analysis of Uplink SCMA With Receiver Diversity and Randomly Deployed Users. IEEE Transactions on Vehicular Technology.

https://doi.org/10.1109/TVT.2017.2728607

\section{Published in:}

IEEE Transactions on Vehicular Technology

\section{Citing this paper}

Please note that where the full-text provided on Manchester Research Explorer is the Author Accepted Manuscript or Proof version this may differ from the final Published version. If citing, it is advised that you check and use the publisher's definitive version.

\section{General rights}

Copyright and moral rights for the publications made accessible in the Research Explorer are retained by the authors and/or other copyright owners and it is a condition of accessing publications that users recognise and abide by the legal requirements associated with these rights.

\section{Takedown policy}

If you believe that this document breaches copyright please refer to the University of Manchester's Takedown Procedures [http://man.ac.uk/04Y6Bo] or contact uml.scholarlycommunications@manchester.ac.uk providing relevant details, so we can investigate your claim.

\section{OPEN ACCESS}




\title{
Performance Analysis of Uplink SCMA with Receiver Diversity and Randomly Deployed Users
}

\author{
Jinchen Bao, Zheng Ma, Member, IEEE, Ming Xiao, Senior Member, IEEE, Zhiguo Ding, Senior Member, IEEE, \\ and Zhongliang Zhu
}

\begin{abstract}
This paper considers the performance analysis of sparse code multiple access (SCMA) with receive diversity arrays and randomly deployed users in a cellular uplink scenario. The impact of path-loss on the performance of SCMA is characterized, by assuming independent Rayleigh fading and joint maximum likelihood (ML) receivers. A tight upper bound on the probability of symbol detection error is derived, and the achievable diversity and coding gains are investigated. The analytical results are validated by using simulations, and show that a diversity order which is equal to the product of the number of receive antennas and the signal-space diversity can be achieved, and the large-scale path-loss decreases only the coding gain.
\end{abstract}

Index Terms-Sparse code multiple access (SCMA), maximum likelihood (ML) detection, randomly deployed users.

\section{INTRODUCTION}

$\mathbf{S}$ PARSE code multiple access (SCMA) techniques have attracted significant interests in academia and industry, and have been proposed for the fifth generation $(5 \mathrm{G})$ wireless networks [1]-[2]. Many aspects relating SCMA have been studied recently, typical examples including the multiuser detection design [3] [4], multidimensional codebooks construction [5]. However, the research works on the performance analysis of SCMA are very limited. This is partially because it is difficult to deal with the multidimensional sparse constellations involved in the multiuser scenarios. In particular, the error performance of uplink and downlink SCMA with maximum likelihood (ML) multiuser detection was analyzed in [6], and a similar analysis was presented in [7] for uplink SCMA with multiple receive antennas. However, only small-scale Rayleigh fadings were investigated.

This work is an extension of [6] to a large-scale fading environment, and the aim is to characterize the impact of path-loss on the performance of SCMA. In particular,

Copyright (c) 2017 IEEE. Personal use of this material is permitted. However, permission to use this material for any other purposes must be obtained from the IEEE by sending a request to pubs-permissions@ieee.org.

The work of J. Bao, Z. Ma and Z. Zhu was supported by Huawei HIRP project (no. YB201504), National Nature Science Foundation of China under Grant 61571373, Key International Cooperation Project of Sichuan Province (no. 2017HH0002), NSFC China-Swedish project (no. 6161101297). The work of M. Xiao was supported by the EU Marie Curie Project, "QUICK", and the work of Z. Ding was supported by the UK EPSRC under grant number EP/L025272/1 and by H2020-MSCA-RISE-2015 under grant number 690750 .

J. Bao, Z. Ma, and Z. Zhu are with the Provincial Key Lab of Information Coding and Transmission, Southwest Jiaotong University, P. R. China (e-mails: jinchen_bao@my.swjtu.edu.cn; zma@home.swjtu.edu.cn; jczuoyi@163.com).

M. Xiao is with the Department of Communication Theory, Royal Institute of Technology (KTH), Stockholm 10044, Sweden (e-mail: mingx@kth.se).

Z. Ding is with the School of Computing and Communications, Lancaster University, LA1 4YW, U.K (e-mail: z.ding@lancaster.ac.uk). the error performance of SCMA with receiving diversity is investigated in an uplink cellular network with randomly deployed users. Two types of situations are considered: the case in which the distances between the users and the base station (BS) are fixed, and the case that the distances are randomly distributed while they are constants within the period of a multidimensional symbol. A tight union bound on the average symbol error rate (SER) under joint ML detection is developed, by assuming perfect channel state information at the receiver and independent Rayleigh fading, which is utilized to show the performance of SCMA. Moreover, the achievable diversity and coding gains are also investigated based on the asymptotic study of pairwise error probability (PEP), which give us insights on how to design power allocation strategies or multiuser codebooks efficiently. Computer simulations are provided to demonstrate the accuracy of the SER analysis and the efficiency of the proposed power allocations.

Notations: The operations $[\cdot]^{t},[\cdot]^{H}$ and $\|\cdot\|$ denote transpose, conjugate transpose and Euclidean norm, respectively, $\mathcal{C N}(\boldsymbol{\mu}, \boldsymbol{\Sigma}) / \mathcal{N}(\boldsymbol{\mu}, \boldsymbol{\Sigma})$ denotes the probability density function (PDF) of complex/real Gaussian random vectors or variables (RVs) with mean $\boldsymbol{\mu}$ and variance $\boldsymbol{\Sigma}$. The form $\operatorname{diag}(\mathbf{h})$ represents a diagonal matrix whose $k$-th diagonal element is from the $k$-th entry of vector $\mathbf{h}, \mathbb{E}[\cdot]$ and $\Re\{\cdot\}$ denote the mean and the real part, respectively, and $\operatorname{Vec}[S]$ denotes a column vector whose entries are chosen from the set $S$ orderly. The identity matrix is denoted by $\mathbf{I}$, and $i=\sqrt{-1}$.

\section{SySTEM MODEL}

We consider a single-cell uplink transmission scenario in which $J$ users/layers and a BS are deployed within a cell, where each user employs only one SCMA layer. The BS, located at the center of the cell, is equipped with $N$ receive antennas and the antenna elements are spaced far enough to ensure independent path-loss, and the users with a single antenna are uniformly distributed within the cell, as shown in Fig. 1. The message from the $j$-th user is mapped into a $K$-dimensional symbol, denoted as $\mathbf{x}_{j}$, and $\mathbf{x}_{j}=\left[x_{j}[1], \cdots, x_{j}[K]\right]^{t}$, which is transmitted over $K$ independent channels, e.g. orthogonal frequency division multiplexing (OFDM) tones. The channels between the $J$ users and the BS are denoted by the $N K \times K J$ channel gain matrix

$$
\mathbf{H}=\left[\begin{array}{ccc}
\operatorname{diag}\left(\mathbf{h}_{1,1}\right) & \cdots & \operatorname{diag}\left(\mathbf{h}_{J, 1}\right) \\
\vdots & \ddots & \vdots \\
\operatorname{diag}\left(\mathbf{h}_{1, N}\right) & \cdots & \operatorname{diag}\left(\mathbf{h}_{J, N}\right)
\end{array}\right]
$$




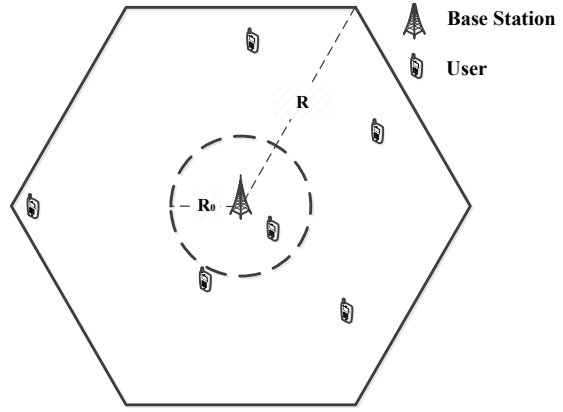

Fig. 1. System model of uplink SCMA with randomly deployed users.

where $\mathbf{h}_{j, n}=\left[h_{j, n}[1], \cdots, h_{j, n}[K]\right]^{t}$, is the $K$-channel vector between the $j$-user and the $n$-th antenna of the BS. Its component satisfies $h_{j, n}[k]=g_{j, n}[k] / d_{j}^{\frac{\alpha}{2}}$, where $g_{j, n}[k] \sim \mathcal{C N}(0,1)$, represents a Rayleigh fading coefficient, $d_{j}$ is the distance between the BS and the $j$-th user, and $\alpha$ is the path-loss exponent. Here we assume that the distance remains constant during a $K$-dimensional symbol period. The path-loss model assumes that the received power decays as [8]

$$
d_{j}^{-\alpha}=\left\{\begin{array}{ll}
R_{0}^{-\alpha}, & d_{j} \leq R_{0} \\
d^{-\alpha}, & R_{0}<d_{j} \leq R
\end{array},\right.
$$

where $R$ and $R_{0}\left(R_{0} \geq 1\right)$ are the maximum and minimum degradation, respectively, $d$ is a RV denoting the distance.

Assume that the multiuser transmissions are synchronized such that the signals for all the users arrive the BS simultaneously. The observed signal vector at the BS is given by

$$
\mathbf{y}=\sum_{j=1}^{J} \sqrt{p_{j} E_{s}} \mathbf{H}_{j} \mathbf{x}_{j}+\mathbf{n}=\sqrt{E_{s}} \mathbf{H} \mathbf{X}+\mathbf{n}
$$

where $E_{s}$ is the total transmitting power for $J$ users, $p_{j}$ is the power allocation coefficient satisfying $\sum_{j=1}^{J} p_{j}=1, \mathbf{H}_{j}=$ $\left[\operatorname{diag}\left(\mathbf{h}_{j, 1}\right), \cdots, \operatorname{diag}\left(\mathbf{h}_{j, N}\right)\right]^{t}$, is the channel corresponding to the $j$-th user, $\mathbf{X}=\left[\sqrt{p_{1}} \mathbf{x}_{1}^{t}, \cdots, \sqrt{p_{J}} \mathbf{x}_{J}^{t}\right]^{t}$, is the combined codeword for $J$ users. The additive white Gaussian noise (AWGN) vector, with independent and identically distributed (i.i.d.) components, is denoted as $\mathbf{n}, \mathbf{n} \sim \mathcal{C N}\left(\mathbf{0}, N_{0} \mathbf{I}\right)$. Therefore, the multiple access channel can be viewed as an equivalent linear system.

Let $\phi_{k}=\left\{j \mid 1 \leq j \leq J, x_{j}[k] \neq 0\right\}$, which denotes the index of users that transmitting over the $k$-th channel. With perfect channel estimation at the BS, the Euclidean distance metric of the joint $\mathrm{ML}$ detector for $\mathbf{X}$ is given by

$$
\hat{\mathbf{X}}=\arg \min _{\mathbf{X}}\left\|\mathbf{y}-\sqrt{E_{s}} \mathbf{H} \mathbf{X}\right\| .
$$

\section{ERror Probability ANALYSIS}

Assume that the codebook size is $C$ and equiprobable transmission for each user is realized, the union bound on the SER for the $j$-th user is given by [6]

$$
P_{j}(e) \leq \frac{1}{C^{J}} \sum_{\mathbf{X}} \sum_{\hat{\mathbf{X}}, \mathbf{x}_{j} \neq \hat{\mathbf{x}}_{j}} P\{\mathbf{X} \rightarrow \hat{\mathbf{X}}\},
$$

where $P\{\mathbf{X} \rightarrow \hat{\mathbf{X}}\}$ is the PEP that the detector mistakes $\mathbf{X}$ with $\hat{\mathbf{X}}$, and $\hat{\mathbf{x}}_{j}$ is the symbol component for the $j$-th user corresponding to $\hat{\mathbf{X}}$.
We first define the following column vectors

$$
\begin{aligned}
\mathbf{x}[k] & =\operatorname{Vec}\left[\left\{\sqrt{p_{j}} x_{j}[k] \mid j \in \phi_{k}\right\}\right], \\
\mathbf{h}_{n}[k] & =\operatorname{Vec}\left[\left\{h_{j, n}[k] \mid j \in \phi_{k}\right\}\right], \\
\mathbf{g}_{n}[k] & =\operatorname{Vec}\left[\left\{g_{j, n}[k] \mid j \in \phi_{k}\right\}\right], \\
\mathbf{d}[k] & =\operatorname{Vec}\left[\left\{d_{j} \mid j \in \phi_{k}\right\}\right] .
\end{aligned}
$$

In the following, we will determine the PEP according to two types of $d_{j}$ :

a) Case I: users with fixed distances such that $d_{j}$ remain constants all the time;

b) Case II: the users are randomly distributed within the cell such that $d_{j}$ are i.i.d. RVs, while $d_{j}$ remain constants during a $K$-dimensional symbol period.

\section{A. Case I: Users with Fixed Distances}

For the linear system in (3), the PEP between $\hat{\mathbf{X}}$ and $\mathbf{X}$ can be calculated as [9]

$$
P\{\mathbf{X} \rightarrow \hat{\mathbf{X}}\}=\operatorname{Pr}\{\Delta<0\}=\frac{1}{2 \pi i} \int_{c-i \infty}^{c+i \infty} \Phi_{\Delta}(s) \frac{\mathrm{d} s}{s},
$$

where $c$ is a positive number to move the integration path away from the singularity at $s=0$,

$$
\begin{aligned}
\Delta & =\left\|\mathbf{y}-\sqrt{E_{s}} \mathbf{H} \hat{\mathbf{X}}\right\|^{2}-\left\|\mathbf{y}-\sqrt{E_{s}} \mathbf{H X}\right\|^{2} \\
& =E_{s}\|\mathbf{H}(\mathbf{X}-\hat{\mathbf{X}})\|^{2}+2 \sqrt{E_{s}} \Re\left\{\mathbf{n}^{H} \mathbf{H}(\mathbf{X}-\hat{\mathbf{X}})\right\},
\end{aligned}
$$

and $\Phi_{\Delta}(s)$ is the Laplace transform of the PDF of $\Delta$. Note that $\Delta$ is a real Gaussian RV conditioned on $\mathbf{H}$, namely $\Delta \sim$ $\mathcal{N}\left(\mu E_{s}, 2 \mu E_{s} N_{0}\right)$ with $\mu=\|\mathbf{H}(\mathbf{X}-\hat{\mathbf{X}})\|^{2}$, then

$$
\Phi_{\Delta}(s)=\mathbb{E}_{\Delta, \mathbf{H}}\left[e^{-s \Delta}\right]=\mathbb{E}_{\mathbf{H}}\left[e^{-s\left(1-s N_{0}\right) E_{s} \mu}\right] .
$$

In (9), $\mu$ can be further expressed as

$$
\begin{aligned}
\mu & =\sum_{n=1}^{N} \sum_{k=1}^{K}\left|\mathbf{h}_{n}[k]^{t}(\mathbf{x}[k]-\hat{\mathbf{x}}[k])\right|^{2} \\
& =\sum_{n=1}^{N} \sum_{k=1}^{K}|\mathbf{g}_{n}[k]^{t} \underbrace{\operatorname{diag}(\mathbf{d}[k])^{-\frac{\alpha}{2}}(\mathbf{x}[k]-\hat{\mathbf{x}}[k])}_{\mathbf{e}[k]}|^{2} \\
& =\sum_{n=1}^{N} \sum_{k=1}^{K} \rho_{k, n},
\end{aligned}
$$

where $\rho_{k, n}=\left|\mathbf{g}_{n}[k]^{t} \mathbf{e}[k]\right|^{2}$. Let

$$
\tau_{k}=\|\mathbf{e}[k]\|^{2}=\sum_{j \in \phi_{k}} p_{j} d_{j}^{-\alpha}\left|x_{j}[k]-\hat{x}_{j}[k]\right|^{2} .
$$

Since $\mathbf{g}_{n}[k] \sim \mathcal{C N}(\mathbf{0}, \mathbf{I}), \mathbf{g}_{n}[k]^{t} \mathbf{e}[k] \sim \mathcal{C N}\left(0, \tau_{k}\right)$. So, $\rho_{k, n}$ are independent exponential RVs with mean $\tau_{k}$. Therefore,

$$
\begin{aligned}
\Phi_{\Delta}(s) & =\mathbb{E}_{\rho_{1,1}, \cdots, \rho_{K, N}}\left[e^{-s\left(1-s N_{0}\right) E_{s} \sum_{n=1}^{N} \sum_{k=1}^{K} \rho_{k, n}}\right] \\
& =\prod_{n=1}^{N} \prod_{k=1}^{K} \mathbb{E}_{\rho_{k, n}}\left[e^{-s\left(1-s N_{0}\right) E_{s} \rho_{k, n}}\right] \\
& =\prod_{k=1}^{K} \frac{1}{\left[1+s\left(1-s N_{0}\right) E_{s} \tau_{k}\right]^{N}},
\end{aligned}
$$

and then, the PEP is given by

$$
P\{\mathbf{X} \rightarrow \hat{\mathbf{X}}\}=\frac{1}{2 \pi i} \int_{c-i \infty}^{c+i \infty} \prod_{k=1}^{K} \frac{1}{\left[1+s\left(1-s N_{0}\right) E_{s} \tau_{k}\right]^{N}} \frac{\mathrm{d} s}{s} .
$$


To investigate the diversity order, we analyze the asymptotic performance using a Chernoff bound estimation for the PEP, which also gives us insights on the performance improvement. The Chernoff bound on the PEP is given by [9]

$$
P_{\mathrm{ch}}\{\mathbf{X} \rightarrow \hat{\mathbf{X}}\}=\min _{q>0} \Phi_{\Delta}(q),
$$

where $q$ is a real variable. Minimizing $\Phi_{\Delta}(q)$ over $q$ is equivalent to maximize $\Psi(q)=\prod_{k=1}^{K}\left[1+q\left(1-q N_{0}\right) E_{s} \tau_{k}\right]$, whose derivative over $q$ is given by

$$
\frac{\mathrm{d} \Psi(q)}{\mathrm{d} q}=\left(1-2 q N_{0}\right) E_{s} \sum_{k=1}^{K} \tau_{k} \prod_{j=1, j \neq q}^{K}\left[1+q\left(1-q N_{0}\right) E_{s} \tau_{j}\right] .
$$

Obviously, $q=\frac{1}{2 N_{0}}$ leads to a local maximum of $\Psi(q)$ and hence a local minimum of $\Phi_{\Delta}(q)$. Note that $\Phi_{\Delta}(q)$ is a positive convex function whose minimum is unique [10], then,

$$
P_{\mathrm{ch}}\{\mathbf{X} \rightarrow \hat{\mathbf{X}}\}=\Phi_{\Delta}\left(\frac{1}{2 N_{0}}\right)=\prod_{k=1}^{K}\left(1+\frac{E_{s}}{4 N_{0}} \tau_{k}\right)^{-N} .
$$

At large signal to noise ratio (SNR) where $\frac{E_{s}}{4 N_{0}} \tau_{k} \gg 1$, by using the approximation $\left(1+\frac{E_{s}}{4 N_{0}} \tau_{k}\right)^{-1} \approx\left(\frac{E_{s}}{4 N_{0}} \tau_{k}\right)^{-1}$, it holds

$$
P_{\mathrm{ch}}\{\mathbf{X} \rightarrow \hat{\mathbf{X}}\} \approx[\frac{E_{s}}{4 N_{0}} \underbrace{\left(\prod_{k=1, \tau_{k} \neq 0}^{K} \tau_{k}\right)^{\frac{1}{\tilde{G}_{d}(\mathbf{X}, \hat{\mathbf{X}})}}}_{\tilde{G}_{c}(\mathbf{X}, \hat{\mathbf{X}})}]^{-N \tilde{G}_{d}(\mathbf{X}, \hat{\mathbf{X}})},
$$

where $\tilde{G}_{d}(\mathbf{X}, \hat{\mathbf{X}})$ is the number of nonzeros in $\tau_{1}, \cdots, \tau_{K}$.

Let $G_{d}$ and $G_{c}$ be the minimum of $\tilde{G}_{d}(\mathbf{X}, \hat{\mathbf{X}})$ and $\tilde{G}_{c}(\mathbf{X}, \hat{\mathbf{X}})$ over all the codeword pairs $\mathbf{X}$ and $\hat{\mathbf{X}}$, respectively. Then, all the PEPs decay with the slope of $\left(E_{s} / N_{0}\right)^{-N G_{d}}$ and the system achieves a diversity order $N G_{d}$. The diversity is in two-fold: the $N$ receiving antennas contribute a diversity of $N$, and $G_{d}$ is the signal-space diversity coming from the use of the multidimensional constellation [11]. Due to the sparness of the codebooks, the signal-space diversity is always less than $K$. Compared with the small-scale fading where $d_{j}=R_{0}$, the large-scale path-loss preserves the diversity but reduces $G_{c}$, which is generally referred to as the coding gain [12].

In general, the nearest neighbours among the multiuser codeword pair $(\mathbf{X}, \hat{\mathbf{X}})$ dominate the performance in the large SNR region, and they correspond to the maximum PEP and hence the minimum ${ }^{1}$ of $\tilde{G}_{c}(\mathbf{X}, \hat{\mathbf{X}})$ and $\tilde{G}_{d}(\mathbf{X}, \hat{\mathbf{X}})$. So, $G_{c}$ and $G_{d}$ are achieved when $\mathbf{X}$ and $\hat{\mathbf{X}}$ are the nearest codewords to each other, for which only one user's symbol pair corresponding to $\mathbf{X}$ and $\hat{\mathbf{X}}$ are different. If only $\mathbf{x}_{j}$ and $\hat{\mathbf{x}}_{j}$ are distinct in the multiuser codeword pair $(\mathbf{X}, \hat{\mathbf{X}})$,

$$
\tau_{k}=p_{j} d_{j}^{-\alpha}\left|x_{j}[k]-\hat{x}_{j}[k]\right|^{2},
$$

and $\tilde{G}_{d}(\mathbf{X}, \hat{\mathbf{X}})=\tilde{w}_{d}\left(\mathbf{x}_{j}, \hat{\mathbf{x}}_{j}\right)$, where $\tilde{w}_{d}\left(\mathbf{x}_{j}, \hat{\mathbf{x}}_{j}\right)$ is the Hamming distance between the multidimensional symbols $\mathbf{x}_{j}$ and $\hat{\mathbf{x}}_{j}$. Then

$$
\tilde{G}_{c}(\mathbf{X}, \hat{\mathbf{X}})=p_{j} d_{j}^{-\alpha} \underbrace{\left(\prod_{k=1, x_{j}[k] \neq \hat{x}_{j}[k]}^{K}\left|x_{j}[k]-\hat{x}_{j}[k]\right|^{2}\right)^{1 / \tilde{w}_{d}\left(\mathbf{x}_{j}, \hat{\mathbf{x}}_{j}\right)}}_{\tilde{w}_{c}\left(\mathbf{x}_{j}, \hat{\mathbf{x}}_{j}\right)} .
$$

${ }^{1}$ This is an approximated minimum for $\tilde{G}_{c}(\mathbf{X}, \hat{\mathbf{X}})$.
Therefore, by minimizing over all the users $(j)$ and all symbol pairs $\left(\mathbf{x}_{j}, \hat{\mathbf{x}}_{j}\right)$, it holds that

$$
\begin{aligned}
G_{d} & =\min _{\forall \mathbf{X} \neq \hat{\mathbf{X}}} \tilde{G}_{d}(\mathbf{X}, \hat{\mathbf{X}})=\min _{j} w_{d, j}, \\
G_{c} & =\min _{\forall \mathbf{X} \neq \hat{\mathbf{X}}} \tilde{G}_{c}(\mathbf{X}, \hat{\mathbf{X}}) \approx \min _{j} \min _{\forall \mathbf{x}_{j} \neq \hat{\mathbf{x}}_{j}} p_{j} d_{j}^{-\alpha} \tilde{w}_{c}\left(\mathbf{x}_{j}, \hat{\mathbf{x}}_{j}\right) \\
& =\min \left\{p_{1} d_{1}^{-\alpha} w_{c, 1}, \cdots, p_{J} d_{J}^{-\alpha} w_{c, J}\right\},
\end{aligned}
$$

where

$$
\begin{aligned}
& w_{c, j}=\min _{\forall \mathbf{x}_{j} \neq \hat{\mathbf{x}}_{j}} \tilde{w}_{c}\left(\mathbf{x}_{j}, \hat{\mathbf{x}}_{j}\right), \\
& w_{d, j}=\min _{\forall \mathbf{x}_{j} \neq \hat{\mathbf{x}}_{j}} \tilde{w}_{d}\left(\mathbf{x}_{j}, \hat{\mathbf{x}}_{j}\right),
\end{aligned}
$$

which are the normalized minimum product distance and the minimum Hamming distance for the $j$-th user's codebook, respectively [11]. It is implied in (20) that the performance of SCMA is dominated by the individual user's codebook with the worst performance.

To improve the performance of SCMA, a useful guidance is to optimize the power allocation strategy or the multiuser codebooks, such that the coding gain and the signal-space diversity are maximized. We take the power allocation as an example. Under the condition of $\sum_{j=1}^{J} p_{j}=1, G_{c}$ is maximized when ${ }^{2}$

$$
p_{1} d_{1}^{-\alpha} w_{c, 1}=\cdots=p_{J} d_{J}^{-\alpha} w_{c, J} .
$$

In most cases, the multiuser codebooks are built from a common mother constellation [1] such that their fundamental properties are the same, then $w_{c, j}$ and $w_{d, j}$ are the same for all the codebooks. Therefore, from $\sum_{j=1}^{J} p_{j}=1$, it is easy to get that

$$
p_{j}=\frac{d_{j}^{\alpha}}{\sum_{j=1}^{J} d_{j}^{\alpha}}, \quad \text { for } j=1, \cdots, J .
$$

Obviously, $p_{j}$ is inversely proportional to the power atenuation, and the solution also assures the fairness among the multiple users in terms of achievable error performance. As for the codebooks optimization, a reliable criterion is to maximize the signal-space diversity $\left(w_{d, j}\right)$ and the minimum product distance $\left(w_{c, j}\right)$ of the mother constellation. Such a problem has been well solved by the linear constellation precoding scheme [14] [15], where unitary transformations can be used over a given mother constellation to increase both the diversity and coding gains.

\section{B. Case II: Users with Random Distances}

In this case, the PDF of the distance variable $d$ in (2) is given by [16]

$$
f_{D}(d)=\left\{\begin{array}{lc}
\frac{2 \pi d}{Q}, & R_{0} \leq d \leq \frac{\sqrt{3} R}{2} \\
\frac{6 d}{Q}\left[\frac{\pi}{3}-2 \arccos \left(\frac{\sqrt{3} R}{2 d}\right)\right], & \frac{\sqrt{3} R}{2} \leq d \leq R
\end{array}\right.
$$

where $Q=3 \sqrt{3} R^{2} / 2-\pi R_{0}^{2}$. Then, the RV $\mu$ in (9) has a different distribution as that in Case I. Let's define the parameter

$$
\hat{\lambda}_{k}=\|\mathbf{x}[k]-\hat{\mathbf{x}}[k]\|^{2}=\sum_{j \in \phi_{k}} p_{j}\left|x_{j}[k]-\hat{x}_{j}[k]\right|^{2},
$$

\footnotetext{
${ }^{2}$ This is similar to the concatenated spherical codes [13], where the optimal concatenation satisfies that the scaled minimum distances are the same for all the component codes. The proof is given in Lemma 3.6 of [13].
} 
and the unitary vector $\mathbf{f}[k]=\frac{1}{\sqrt{\hat{\lambda}_{k}}}(\mathbf{x}[k]-\hat{\mathbf{x}}[k])$. Then, $\mu$ can be expressed as

$$
\begin{aligned}
\mu & =\sum_{n=1}^{N} \sum_{k=1}^{K}\left|\mathbf{h}_{n}[k]^{t}(\mathbf{x}[k]-\hat{\mathbf{x}}[k])\right|^{2} \\
& =\sum_{n=1}^{N} \sum_{k=1}^{K} \hat{\lambda}_{k}\left|\mathbf{h}_{n}[k]^{t} \mathbf{f}[k]\right|^{2} .
\end{aligned}
$$

It is difficult to determine the distribution of $\left|\mathbf{h}_{n}[k]^{t} \mathbf{f}[k]\right|^{2}$, since $\mathbf{h}_{n}[k]^{t} \mathbf{f}[k]$ is a weighted sum of several i.i.d. RVs. As $\mathbf{f}[k]$ is unitary, we assume that $\mathbf{h}_{n}[k]^{t} \mathbf{f}[k]$ has the same distribution as the elements in $\mathbf{h}_{n}[k]$. The assumption is based on the fact that the performance is dominated by the PEPs of nearest neighbors of codewords, it corresponds to the smallest multiuser distances and for which a very small number of users are involved in $\mathbf{f}[k]$. With joint detection, the approximation becomes more accurate in the large SNR region. Since in this case, the number of errorly detected users is small and if only one user is in error, there will be only one nonzero element in $\mathbf{f}[k]$ and the assumption comes true. Then, we assume $\mathbf{h}_{n}[k]^{t} \mathbf{f}[k] \approx \tilde{g}_{n, k} \tilde{d}^{-\frac{\alpha}{2}}$, where $\tilde{g}_{n, k} \sim \mathcal{C N}(0,1)$ and $\tilde{d}$ has the same distribution as $d_{j}$. Thus,

$$
\mu \approx \tilde{d}^{-\alpha} \underbrace{\sum_{n=1}^{N} \sum_{k=1}^{K} \hat{\lambda}_{k}\left|\tilde{g}_{n, k}\right|^{2}}_{V}=\tilde{d}^{-\alpha} V .
$$

In the following, we determine the PDF of $\mu$. Let $\boldsymbol{\lambda}=$ $\left[\lambda_{1}, \cdots, \lambda_{M}\right]^{t}$, denoting the $M(M \leq K)$ distinct values in the set $\left\{\hat{\lambda}_{1}, \cdots, \hat{\lambda}_{K}\right\}$, and $\mathbf{r}=\left[r_{1}, \cdots, r_{M}\right]^{t}$, denoting the number of entries in $\left\{\hat{\lambda}_{1}, \cdots, \hat{\lambda}_{K}\right\}$ that are equal to $\lambda_{1}, \cdots, \lambda_{M}$ and times $N$, respectively. Since $\tilde{g}_{n, k} \sim \mathcal{C N}(0,1), V$ is a weighted sum of $N K$ exponential RVs, which is generalized Chi-squared distributed with PDF given by [6][17]

$$
f_{V}(v)=A_{\mathbf{r}, \boldsymbol{\lambda}} \sum_{m=1}^{M} \sum_{l=1}^{r_{m}} \frac{B_{m, l, \mathbf{r}, \boldsymbol{\lambda}}}{\left(r_{m}-l\right) !} v^{r_{m}-l} e^{-\frac{v}{\lambda_{m}}}
$$

where $A_{\mathbf{r}, \boldsymbol{\lambda}}=\prod_{m=1}^{M} \lambda_{m}^{-r_{m}}$ and

$$
\begin{aligned}
& B_{m, l, \mathbf{r}, \boldsymbol{\lambda}}=(-1)^{l+1} \sum_{\eta \in \Omega_{m, l}} \prod_{j=1, j \neq m}^{M}\left(\begin{array}{c}
\eta_{j}+r_{j}-1 \\
\eta_{j}
\end{array}\right) \\
& \times\left(\frac{1}{\lambda_{j}}-\frac{1}{\lambda_{m}}\right)^{-\left(r_{j}+\eta_{j}\right)},
\end{aligned}
$$

for which $\boldsymbol{\eta}=\left[\eta_{1}, \cdots, \eta_{M}\right]^{t}$, which is chosen from the set $\Omega_{m, l}$ given by

$$
\Omega_{m, l}=\left\{\boldsymbol{\eta} \in \mathbb{Z}^{M} \mid \sum_{j=1}^{M} \eta_{j}=l-1, \eta_{m}=0, \eta_{j} \geq 0 \forall j\right\} .
$$

Similar as that in [16], from (2), (24) and (27), we can derive the PDF of $\mu$ as

$$
\begin{aligned}
f_{\mu}(\mu) & =\frac{4 \pi}{3 \sqrt{3} R^{2}}\left[\frac{R_{0}^{\alpha+2}}{2} f_{V}\left(R_{0}^{\alpha} \mu\right)+\int_{R_{0}}^{R} t^{\alpha+1} f_{V}\left(t^{\alpha} \mu\right) \mathrm{d} t\right. \\
& \left.-\frac{6}{\pi} \int_{\frac{\sqrt{3} R}{2}}^{R} t^{\alpha+1} \arccos \left(\frac{\sqrt{3} R}{2 t}\right) f_{V}\left(t^{\alpha} \mu\right) \mathrm{d} t\right] .
\end{aligned}
$$

Then, the Lapalace transform in (9) is given by

$$
\begin{aligned}
\Phi_{\Delta}(s) & \approx \int_{0}^{\infty} e^{-s\left(1-s N_{0}\right) E_{s} \mu} f_{\mu}(\mu) \mathrm{d} \mu \\
& =\frac{4 \pi}{3 \sqrt{3} R^{2}}\left[\frac{R_{0}^{\alpha+2}}{2} \Upsilon\left(s, R_{0}^{\alpha}\right)+\int_{R_{0}}^{R} t^{\alpha+1} \Upsilon\left(s, t^{\alpha}\right) \mathrm{d} t\right. \\
& \left.-\frac{6}{\pi} \int_{\frac{\sqrt{3} R}{2}}^{R} t^{\alpha+1} \arccos \left(\frac{\sqrt{3} R}{2 t}\right) \Upsilon\left(s, t^{\alpha}\right) \mathrm{d} t\right],
\end{aligned}
$$

where

$$
\begin{aligned}
& \Upsilon(s, a)=\int_{0}^{\infty} e^{-s\left(1-s N_{0}\right) E_{s} \mu} f_{V}(a \mu) \mathrm{d} \mu \\
& =A_{\mathbf{r}, \boldsymbol{\lambda}} \sum_{m=1}^{M} \sum_{l=1}^{r_{m}} \frac{B_{m, l, \mathbf{r}, \boldsymbol{\lambda}}}{\left(r_{m}-l\right) !} \int_{0}^{\infty} e^{-s\left(1-s N_{0}\right) E_{s} \mu}(a \mu)^{r_{m}-l} e^{-\frac{a \mu}{\lambda_{m}}} \mathrm{~d} \mu \\
& =A_{\mathbf{r}, \boldsymbol{\lambda}} \sum_{m=1}^{M} \sum_{L=1}^{r_{m}} \frac{a^{L-1} \lambda_{m}^{L} B_{m, r_{m}-L+1, \mathbf{r}, \boldsymbol{\lambda}}}{\left[s\left(1-s N_{0}\right) E_{s} \lambda_{m}+a\right]^{L}}
\end{aligned}
$$

where the last step of (33) is achieved by calculating the integral and substituting $l$ with $r_{m}-L+1$, and $a$ stands for $R_{0}^{\alpha}$ and $t^{\alpha}$ in (32).

Therefore, by combing (7), (32) and (33), the PEP can be approximately estimated as

$$
\begin{aligned}
P\{\mathbf{X} \rightarrow \hat{\mathbf{X}}\} & \approx \frac{2 A_{\mathbf{r}, \boldsymbol{\lambda}}}{3 \sqrt{3} R^{2} i} \sum_{m=1}^{M} \sum_{L=1}^{r_{m}} \lambda_{m}^{L} B_{m, r_{m}-L+1, \mathbf{r}, \boldsymbol{\lambda}} \\
& \times \int_{c-i \infty}^{c+i \infty}\left[C_{s, \lambda_{m}, L}+D_{s, \lambda_{m}, L}-E_{s, \lambda_{m}, L}\right] \frac{\mathrm{d} s}{s},
\end{aligned}
$$

where

$$
\begin{aligned}
C_{s, \lambda_{m}, L} & =\frac{R_{0}^{\alpha L+2}}{2\left[s\left(1-s N_{0}\right) E_{s} \lambda_{m}+R_{0}^{\alpha}\right]^{L}}, \\
D_{s, \lambda_{m}, L} & =\int_{R_{0}}^{R} \frac{t^{\alpha L+1}}{\left[s\left(1-s N_{0}\right) E_{s} \lambda_{m}+t^{\alpha}\right]^{L}} \mathrm{~d} t, \\
E_{s, \lambda_{m}, L} & =\frac{6}{\pi} \int_{\frac{\sqrt{3} R}{2}}^{R} \frac{t^{\alpha L+1} \arccos \left(\frac{\sqrt{3} R}{2 t}\right)}{\left[s\left(1-s N_{0}\right) E_{s} \lambda_{m}+t^{\alpha}\right]^{L}} \mathrm{~d} t .
\end{aligned}
$$

In the following, we investigate the diversity order for the Case II. Since $\tilde{g}_{n, k} \sim \mathcal{C N}(0,1)$ and hence $\left|\tilde{g}_{n, k}\right|^{2}$ are i.i.d. exponential RVs with unit mean, from (9) and (27), it holds

$$
\begin{aligned}
\Phi_{\Delta}(s) & \approx \mathbb{E}_{\tilde{g}_{1,1}, \cdots, \tilde{g}_{N, K}, \tilde{d}}\left[e^{-s\left(1-s N_{0}\right) E_{s} \tilde{d}^{-\alpha} \sum_{n, k} \hat{\lambda}_{k}\left|\tilde{g}_{n, k}\right|^{2}}\right] \\
& =\mathbb{E}_{\tilde{d}}\left\{\prod_{n=1}^{N} \prod_{k=1}^{K} \mathbb{E}_{\tilde{g}_{n, k}}\left[e^{\left.-s\left(1-s N_{0}\right) E_{s} \tilde{d}^{-\alpha} \hat{\lambda}_{k}\left|\tilde{g}_{n, k}\right|^{2}\right]}\right\}\right. \\
& =\mathbb{E}_{\tilde{d}}\left[\prod_{k=1}^{K} \frac{1}{\left[1+s\left(1-s N_{0}\right) E_{s} \tilde{d}^{-\alpha} \hat{\lambda}_{k}\right]^{N}}\right] .
\end{aligned}
$$

As the distance RV satisfy $0<\tilde{d} \leq R$, similar as that in (16), the Chernoff bound approximation is given by

$$
\begin{aligned}
P_{\mathrm{ch}}\{\mathbf{X} \rightarrow \hat{\mathbf{X}}\} & \approx \mathbb{E}_{\tilde{d}}\left[\prod_{k=1}^{K} \frac{1}{\left(1+\frac{E_{s}}{4 N_{0}} \hat{\lambda}_{k} \tilde{d}^{-\alpha}\right)^{N}}\right] \\
& \stackrel{(a)}{\approx} \mathbb{E}_{\tilde{d}}\left[\prod_{k=1, \hat{\lambda}_{k} \neq 0}^{K}\left(\frac{E_{s}}{4 N_{0}} \hat{\lambda}_{k} \tilde{d}^{-\alpha}\right)^{-N}\right] \\
& =\left[\frac{E_{s}}{4 N_{0}} \tilde{G}_{c}(\mathbf{X}, \hat{\mathbf{X}})\right]^{-N \tilde{G}_{d}(\mathbf{X}, \hat{\mathbf{X}})}
\end{aligned}
$$


TABLE I

SIMULATION PARAMETERS FOR UNCODED/CODED SCMA SCENARIOS

\begin{tabular}{c|c}
\hline \hline Parameters & Values \\
\hline \hline \multirow{3}{*}{ Factor graph matrix } & $\mathbf{F}=\left[\begin{array}{llllll}1 & 0 & 1 & 0 & 1 & 0 \\
0 & 1 & 1 & 0 & 0 & 1 \\
1 & 0 & 0 & 1 & 0 & 1 \\
0 & 1 & 0 & 1 & 1 & 0\end{array}\right]$ \\
& Uncoded, turbo code (code length 1008 bits) \\
\hline Channel coding & 4-ary codebook \\
\hline Interleaver & Random interleaver (interleaving depth 1008 bits) \\
\hline Codebook & uncoded: ML, MPA (6 iterations), \\
\hline \multirow{2}{*}{ Receiver } & coded: turbo-MPA (3 MPA + 3 outer iterations) \\
\hline Channel model & Uplink i.i.d. Rayleigh fading channel \\
\hline \multirow{2}{*}{$d_{j}$ for Case I } & $R=3$ m: $\{2.54,1.36,2.74,2.54,1.88,1.60\} \mathrm{m}$ \\
& $R=5$ m: $\{3.30,2.87,3.71,3.81,2.89,3.51\} \mathrm{m}$ \\
\hline \hline
\end{tabular}

where $(a)$ is from the large SNR approximation as in Case I, $\tilde{G}_{d}(\mathbf{X}, \hat{\mathbf{X}})$ is the number of nonzeros in $\hat{\lambda}_{1}, \cdots, \hat{\lambda}_{K}$, and

$\tilde{G}_{c}(\mathbf{X}, \hat{\mathbf{X}})=\left(\prod_{k=1, \hat{\lambda}_{k} \neq 0}^{K} \hat{\lambda}_{k}\right)^{\frac{1}{\tilde{G}_{d}(\mathbf{X}, \hat{\mathbf{X}})}} \mathbb{E}_{\tilde{d}}\left[\tilde{d}^{\alpha N \tilde{G}_{d}(\mathbf{X}, \hat{\mathbf{X}})}\right]^{-\frac{1}{N \tilde{G}_{d}(\mathbf{X}, \tilde{\mathbf{X}})}}$,

which is a constant that is independent with SNR, denoting the coding advantage. Therefore, the system achieves the same diversity order but a different coding gain with that in Case I. Using a similar analysis as that in Case I, if the fundamental properties of the multiuser codebooks are the same, equal power allocation strategy will be a reasonable choice in terms of achieving good performance for Case II.

\section{Numerical Results and Simulations}

In this section, simulation results are presented to demonstrate the performance of SCMA, and also verify the effectiveness of the analysis. The considered SCMA system follows a factor graph matrix as in Table I, where 6 users multiplexing over 4 spreading chips $(J=6, K=4)$. The performance of both joint ML and message passing algorithm (MPA) detection are investigated, and equal power allocations are assumed. The detailed simulation configurations for uncoded and coded scenarios are given in Table I. Without loss of generality, we set $R_{0}=1.2 \mathrm{~m}$ all the time.

We first provides the results for Case I. Fig. 2 illustrates the diversity gains of the uncoded SCMA scheme with fading parameters $R=3 \mathrm{~m}, \alpha=2$ and $1 / 2 / 3$ receive antennas. First, we can see that the analytical results of the SER analysis are very tight in the large SNR region, for all numbers of receive antennas. Second, it can be seen that a considerable performance improvement can be achieved with diversity arrays. With only two receive antennas, the system provides a huge gain that has an overall diversity order of 4 , where the multidimensional codebook contributes a signal-space diversity of two. As an example for performance enhancement, we also provide the results with the proposed power allocation. It is obvious that the performance are improved and the gains become more evident for a larger number of receive antennas.

Fig. 3 shows the impact of different path-loss parameters on the performance of uncoded SCMA with two receive antennas.

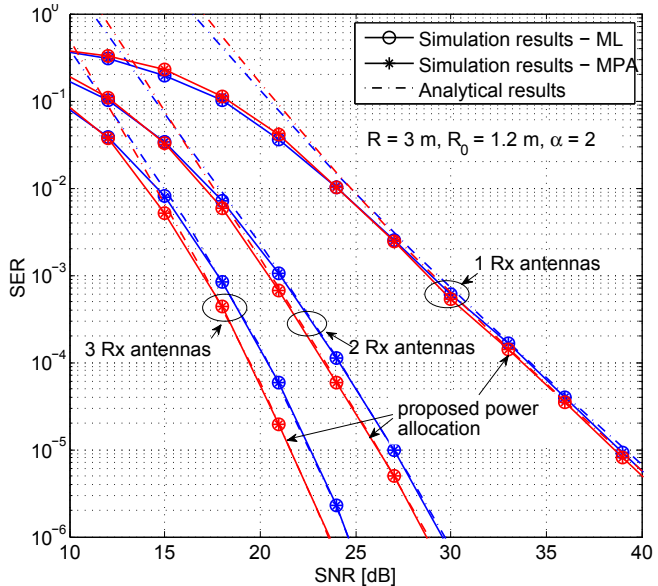

Fig. 2. Illustration of diversity gains of uncoded SCMA in Case I, with $R=3 \mathrm{~m}, R_{0}=1.2 \mathrm{~m}, \alpha=2$. Blue and red curves are for equal and proposed power allocations, respectively.

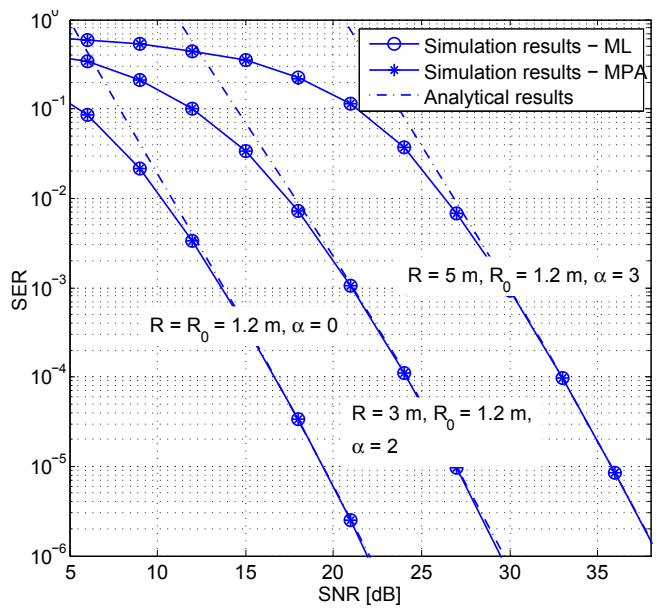

Fig. 3. Impact of path-loss on the performance of uncoded SCMA in Case $\mathrm{I}$, with two receive antennas $(N=2)$.

The result of small-scale fading environment where $\alpha=0$ is also presented for comparison. In this configuration, the diversity order is always four, but there is a very large gap in coding gains for different path-loss environments. Both the distances between the users and the BS and the path-loss factor greatly deteriorate the SER performance, which means that considerable SNRs are required to compensate the power loss for long distance transmissions.

Fig. 4 and 5 present the uncoded results for Case II. In Fig. 4, performance of SCMA with different diversity arrays are provided for randomly distributed users. A similar observation can be observed as that in Fig. 2, a great improvement in SER performance can be achieved with receive diversity. While the theoretical performance analysis is based on the approximated union bound, the analytical bounds are still very tight for all numbers of receive antennas, which shows the effectiveness of the proposed method for SER estimation. Also, Fig. 5 investigates the impact of different path-loss on the performance. Just as expected, the large-scale path-loss preserves the diversity order while decreases the coding gain. Moreover, it is evident that the performance of the MPA detector is as good as the optimal ML detector, for all the 


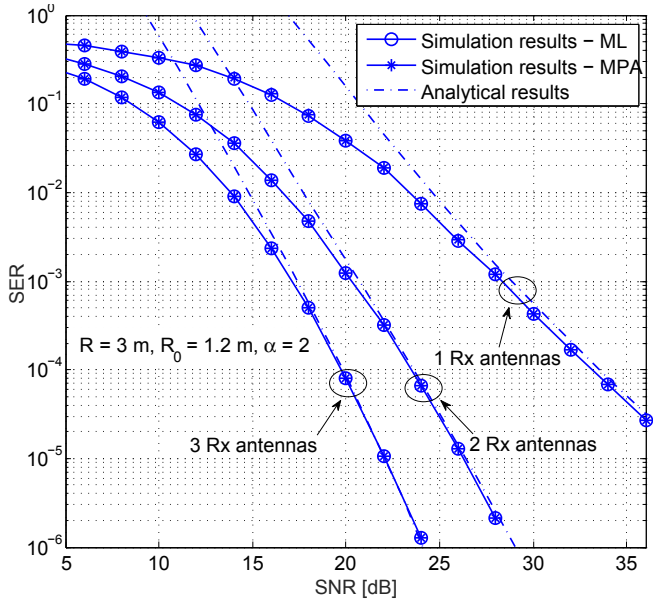

Fig. 4. Illustration of diversity gains of uncoded SCMA in Case II, with $R=3 \mathrm{~m}, R_{0}=1.2 \mathrm{~m}, \alpha=2$.

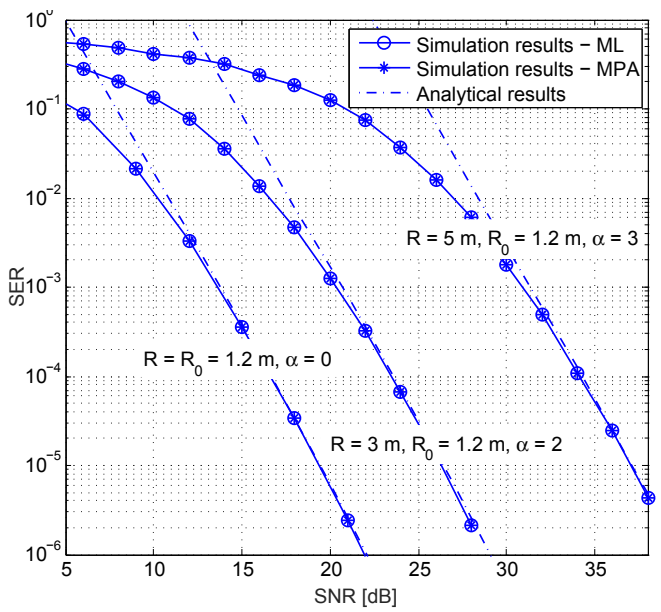

Fig. 5. Impact of path-loss on the performance of uncoded SCMA in Case II, with two receive antennas $(N=2)$.

simulation scenarios and fading parameters.

Finally, to further verify the effectiveness of the proposed power allocations, Fig. 6 provides the simulated bit-error rate (BER) performance of a bit-interleaved half-rate turbo-coded SCMA scenario for Case I, with fading parameters $R=$ $3 \mathrm{~m}, \alpha=2$. An iterative detection and decoding receiver [4] with 3 MPA iterations and 3 outer iterations is employed. As it is observed, a very large performance gain can be achieved with the proposed power allocation strategy. Moreover, the performance gap between the BER curves becomes larger with receiver diversity arrays, which agrees with the results of Fig. 2 for uncoded scenarios.

\section{CONCLUSIONS}

We have carried out in this paper the performance analysis for uplink SCMA scenarios with receiving diversity and randomly distributed users. Tight upper bounds on the SER have been developed for two situations where the distances between the users and the BS are fixed and random. For both cases, it is shown that with only a small number of receive antennas, SCMA can achieve significant SER performance gains. From both theoretical and simulation results, the study shows that a diversity order equals to the product of the number of receive

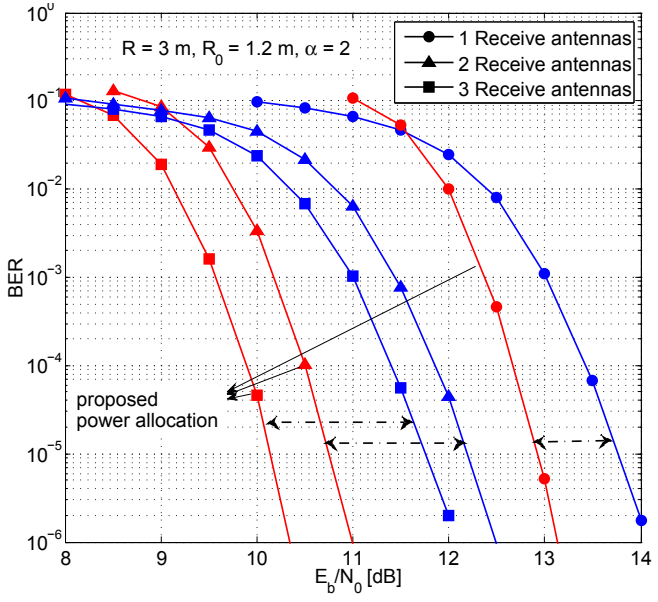

Fig. 6. BER performance of turbo-coded SCMA with receiver diversity under different power allocations in Case I, $R=3 \mathrm{~m}, R_{0}=1.2 \mathrm{~m}, \alpha=2$.

antennas and the signal-space diversity can be achieved, and the large-scale path-loss reduces only the coding gain.

\section{REFERENCES}

[1] H. Nikopour and H. Baligh, "Sparse code multiple access," in Proc. IEEE 24th Int. Symp. Pers., Indoor \& Mobile Radio Commun. (PIMRC'13), London, Sep. 2013, pp. 332-336.

[2] Z. Ding, et.al., "A survey on non-orthogonal multiple access for $5 \mathrm{G}$ networks: Research challenges and future trends," IEEE J. Sel. Areas Commun., vol. PP, no. 99, pp. 1-1, 2017.

[3] F. Wei and W. Chen, "Low complexity iterative receiver design for sparse code multiple access," IEEE Trans. Commun., vol. 65, no. 2, pp. 621634, Feb. 2017

[4] Y. Wu, S. Zhang, and Y. Chen, "Iterative multiuser receiver in sparse code multiple access systems," in Proc. IEEE Int. Commun. Conf. (ICC'15), London, Jun. 2015, pp. 2918-2923.

[5] J. Bao, Z. Ma, Z. Ding. G. K. Karagiannidis, and Z. Zhu, "On the design of multiuser codebooks for uplink SCMA systems," IEEE Commun. Letters, vol. 20, no. 10, pp. 1920-1923, Oct. 2016.

[6] J. Bao, Z. Ma, G. K. Karagiannidis, M. Xiao, and Z. Zhu, "Joint multiuser detection of multidimensional constellations over fading channels," IEEE Trans. Commun., vol. 65, no. 1, pp. 161-172, Jan. 2017.

[7] S. C. Lim, N. Kim, and H. Park, "Uplink SCMA system with multiple antennas," IEEE Trans. Veh. Tech., vol. PP, no. 99, pp. 1-1, 2017.

[8] I. Krikidis, "Simultaneous information and energy transfer in large-scale networks with/without Relaying," IEEE Trans. Commun., vol. 62, no. 3, pp. 900-912, Mar. 2014.

[9] E. Biglieri, et al., "Simple method for evaluating error probabilities," IEE Electron. Letters, vol. 32, no. 3, pp. 191-192, Feb. 1996

[10] S. Furrer and D. Dahlhaus, "Multiple-antenna signaling over fading channels with estimated channel state information: Performance analysis," IEEE Trans. Inf. Theory, vol. 53, no. 6, pp. 2010-2027, Jun. 2007.

[11] J. Boutros and E. Viterbo, "Signal space diversity: A power- and bandwidth-efficient diversity technique for the Rayleigh fading channel," IEEE Trans. Inf. Theroy, vol. 44, no. 4, pp. 1453-1467, July 1998.

[12] Z. Wang and G. B. Giannakis, "A simple and general parameterization quantifying performance in fading channels," IEEE Trans. Commun., vol. 51, no. 8, pp. 1389-1398, Aug. 2003.

[13] J. Hamkins, "Design and analysis of spherical codes," Ph.D. dissertation, Univ. of Illinois at Urbana-Champaign, Sep. 1996.

[14] Y. Xin, Z. Wang, and G. B. Giannakis, "Space-time diversity systems based on linear constellation precoding," IEEE Trans. Wireless Commun., vol. 2, no. 2, pp. 294-309, Mar. 2003.

[15] Z. Liu, Y. Xin, and G. B. Giannakis, "Linear constellation precoding for OFDM with maximum multipath diversity and coding gains," IEEE Trans. Commun., vol. 51, no. 3, pp. 416-427, Mar. 2003.

[16] Z. Yang, X. Lei, Z. Ding, P. Fan, and G. K. Karagiannidis, "On the uplink sum rate of SCMA system with randomly deployed users," IEEE Wireless Commun. Letters, vol. PP, no. 99, pp. 1-1, Mar. 2017.

[17] E. Björnson, et.al., "Exploiting quantized channel norm feedback through conditional statistics in arbitrarily correlated MIMO systems," IEEE Trans. Signal Process., vol. 57, no. 10, pp. 4027-4041, Oct. 2009. 